\title{
Efficient Narrowband Direction of Arrival Estimation Based on a Combination of Uniform Linear/Shirvani-Akbari Arrays
}

\author{
Shahriar Shirvani Moghaddam ${ }^{1}$ and Farida Akbari ${ }^{2}$ \\ ${ }^{1}$ Digital Communications Signal Processing Research Laboratory, Faculty of Electrical and Computer Engineering, \\ Shahid Rajaee Teacher Training University (SRTTU), Lavizan, Tehran 16788-15811, Iran \\ ${ }^{2}$ Electrical Engineering Department, Faculty of Engineering, Tehran South Branch, Islamic Azad University, Ahang Boulevard, \\ Tehran, Iran \\ Correspondence should be addressed to Shahriar Shirvani Moghaddam, sh_shirvani@srttu.edu
}

Received 29 March 2012; Revised 30 July 2012; Accepted 30 July 2012

Academic Editor: Matteo Pastorino

Copyright ( 2012 S. Shirvani Moghaddam and F. Akbari. This is an open access article distributed under the Creative Commons Attribution License, which permits unrestricted use, distribution, and reproduction in any medium, provided the original work is properly cited.

\begin{abstract}
Uniform linear array (ULA) geometry does not perform well for direction of arrival (DOA) estimation at directions close to the array endfires. Shirvani and Akbari solved this problem by displacing two elements from both ends of the ULA to the top and/or bottom of the array axis. Shirvani-Akbari array (SAA) presents a considerable improvement in the DOA estimation of narrowband sources arriving at endfire directions in terms of DOA estimation accuracy and angular resolution. In this paper, all new proposed SAA configurations are modelled and also examined, numerically. In this paper, two well-known DOA estimation algorithms, multiple signal classification (MUSIC) and minimum variance distortionless response (MVDR), are used to evaluate the effectiveness of proposed arrays using total root mean square error (RMSE) criterion. In addition, two new scenarios are proposed which divide angular search to two parts, directions close to array endfires as well as middle angles. For middle angles, which belong to $\left(-70^{\circ} \leq \theta \leq 70^{\circ}\right)$, ULA is considered, and for endfire angles, the angles which belong to $\left(-90^{\circ} \leq \theta \leq-70^{\circ}\right)$ and $\left(70^{\circ} \leq \theta \leq 90^{\circ}\right)$, SAA is considered. Simulation results of new proposed scenarios for DOA estimation of narrowband signals show the better performance with lower computational load.
\end{abstract}

\section{Introduction}

Rapid growth of requests for mobile communications and increasing operators' demands has stimulated considerable studies for development of advanced techniques and technologies. The goal is to manage limited radio resources such as spectrum and power and providing fully integrated, low cost, reliable, high quality, and high speed wireless networks. In recent years, smart antennas or adaptive array antennas were introduced as effective solutions for these growing demands [1-3]. Smart antennas exploit array processing techniques such as adaptive beamforming and parameter estimation methods to improve the system performance.

Array processing is generally a useful field of signal processing that is utilized in different engineering applications, for measurement of propagation fields such as mechanical, acoustic, and radio waves. Signal sources can be detected using an array of sensors, and many of the signal parameters including directivity, frequency, polarization, and other various information can be extracted using array processing. Direction of arrival (DOA) is one of the most important parameters that is considered specially in wireless communications and smart antennas to increase the system efficiency [4]. In this paper, the focus will be on DOA estimation of the narrowband radio waves for smart antenna applications. However, the proposed arrays and also efficient combination of arrays can be used in the other fields of array processing.

The main algorithms often used in the smart antenna systems are beamforming and DOA estimation algorithms which are performed in a digital signal processing unit. Beamforming algorithms dynamically adjust the radiation pattern to emphasize signals of interest (SOIs) and to minimize undesired interferers or signals not of interest (SNOIs). 
Higher capacity and data rate, lower power consumption, increased coverage range, better quality of service, reduction in noise and fading effects, and suppression of interferers are advantages of employing adaptive array antennas [1-3].

DOA estimation algorithms are used to determine the direction of received or transmitted signals in order to help the beamforming process and spatial filtering which reduce the interference or multipath fading effects. DOA estimation methods may also be employed for detection and tracking of moving signal sources, characterization and modelling of spatial/temporal channels, and presenting location-based services in mobile networks. Therefore, DOA estimation is an important research area in wireless communications. Numerous algorithms are available and a large number of modified algorithms and enhancement techniques are proposed in the literatures $[4,5]$. Although DOA estimation is not a new issue and many of the algorithms have been proposed several years ago, it is still interesting and many research works are accomplished in this area. Superresolution and Multidimensional (MD) DOA estimation are considerable topics in today wireless communication investigations [6]. In many applications, it is necessary to extend one-dimensional (1D) DOA estimation to twodimensional (2D). In some problems such as channel estimation or prediction or modelling, or multiinput multioutput (MIMO) communication, jointly determination of the DOA and other signal parameters such as Doppler, polarization, delay, and so forth are required $[5,6]$. Angle estimation in the presence of multipath, interference, source correlation, array miscalibration, coupling effects, and nonuniform noise is widely studied in the literature [6].

The structure of the array is a way to improve the smart antenna performance which has been studied less than smart antenna algorithms. Antenna array consists of a set of antennas, arranged in a particular geometry. Array configuration affects the performance of DOA estimation methods and some of the DOA estimation algorithms can be applied on certain geometries such as linear or circular arrays. Uniform linear array (ULA) is the simplest geometry for analysis and implementation. ULA generates narrow beams and therefore provides appropriate accuracy and resolution during beamforming and DOA estimation. However, ULA cannot analyse more than one dimension. Furthermore, DOA estimation accuracy with the ULA decreases at the angles close to array endfires. Therefore, other array geometries are investigated to obtain better performance. Uniform circular array (UCA) is another common geometry which gives the two-dimensional coverage and uniform performance in all azimuth directions with further complexity than ULA [7]. Some array geometries such as concentric circular arrays (CCAs), hexagonal arrays, and other planar arrays have been examined for DOA estimation and beamforming purposes $[8,9]$. Arbitrary geometries and nonuniform array configurations are also considered in the literature [6]. However, many of these arrangements are complex in calculation and analysis. So, in many research works, it is attempted to develop linear arrays or combine them together to obtain better performance while having proportional simplicity. Parallel linear arrays, displaced sensor arrays (DSAs), uniform rectangular arrays (URAs), and L-shaped, V-shaped, and Y-shaped arrays are some of the investigated configurations [10-16]. These geometries have the capability of 2D-DOA estimation. Many of these arrangements have enhanced DOA estimation accuracy at endfire directions and each of them has particular properties including beamwidth, resolution threshold, computational complexity, insensitivity to the direction and Cramer-Rao Bounds (CRBs).

In this paper, at first it is attempted to mitigate the ULA drawback in endfire directions by displacing two elements of ULA from both ends of the array to different positions in top and/or bottom of the linear array. The proposed arrays performance is evaluated via two different well-known DOA estimation algorithms, namely, multiple signal classification (MUSIC) and minimum variance distortionless response (MVDR). Finally, a new combination of ULA and proposed arrays is proposed through two scenarios which offer lower root mean square error (RMSE) with respect to ULA and PAs in different signal to noise ratios (SNRs) and DOAs.

The rest of the paper is organized as follows. Section 2 presents signal model for the antenna array in the ULA and the new proposed Shirvani-Akbari array (SAA) geometries and corresponding formulations are stated. Section 3 provides a brief overview of DOA estimation methods and describes MUSIC and MVDR algorithms. Simulation results and the performance of different proposed SAA configurations are illustrated in Section 4, and angular spectrums and total RMSE of the estimated DOAs with different array geometries are compared. As the main goal of this paper, Section 5 proposes an appropriate combination of the ULA and different proposed SAAs for calculation the MUSIC and MVDR spectrums and illustrates some numerical results in two scenarios. Finally, Section 6 concludes the paper.

\section{Signal Model and Array Configuration}

Received signals can be expressed as a linear combination of incident signals and zero mean Gaussian noise. The incident narrowband signals are assumed to be uncorrelated direct line of sight (LOS) and also uncorrelated with the noise. The input signal vector denoted by $\mathbf{x}(t)$ can be written as

$$
\mathbf{x}(t)=\sum_{m=1}^{M} \mathbf{a}\left(\theta_{m}\right) s_{m}(t)+\mathbf{n}(t)=\mathbf{A} \mathbf{s}(t)+\mathbf{n}(\mathbf{t})
$$

where $s_{m}(t)$ denotes the $m$ th signal source at direction $\theta_{m}$ from the array boresight. $\mathbf{a}\left(\theta_{m}\right)$ is the $N \times 1$ steering vector or response vector of the array in direction of $\theta_{m}$, where $N$ represents the number of elements. $\mathbf{s}(t)$ denotes the $M \times 1$ vector containing $M$ incident signals on the array. $\mathbf{n}(t)$ is an $M \times 1$ vector containing $M$ uncorrelated additive white Gaussian noises (AWGNs) on the array. A is an $N \times M$ matrix of steering vectors, which is named as manifold matrix as

$$
\mathbf{A}=\left[\mathbf{a}\left(\theta_{1}\right) \mathbf{a}\left(\theta_{2}\right) \cdots \mathbf{a}\left(\theta_{M}\right)\right] .
$$


The spatial correlation matrix $\mathbf{R}_{\mathbf{x x}}$ of the received signal is defined by

$$
\mathbf{R}_{\mathbf{X X}}=E\left[\mathbf{x}(t) \cdot \mathbf{x}^{\mathbf{H}}(t)\right]
$$

where $E[\cdot]$ and $[\cdot]^{H}$ are the expectation and the Hermitian (conjugate transpose) operators, respectively. Substituting (1) into (3), $\mathbf{R}_{\mathbf{x x}}$ can be written as

$$
\mathbf{R}_{\mathbf{x x}}=E\left[\mathbf{A} \cdot \mathbf{s}(t) \cdot \mathbf{s}^{H}(t) \cdot \mathbf{A}^{\mathbf{H}}\right]+E\left[\mathbf{n}(t) \cdot \mathbf{n}^{\mathbf{H}}(t)\right] .
$$

And finally the spatial correlation matrix can be expressed as

$$
\mathbf{R}_{\mathrm{xx}}=\mathbf{A R}_{\mathbf{s s}} \mathbf{A}^{\mathrm{H}}+\sigma_{n}^{2} \mathbf{I} .
$$

$\mathbf{R}_{\mathrm{ss}}$ is the $M \times M$ signal correlation matrix. $\sigma_{n}^{2}$ and I denote variance of the noise and identity matrix, respectively. Since the antennas cannot receive DC signals, the mean values of arriving signals and noise are zero and so the correlation matrix obtained in (5) is referred to the covariance matrix [11]. This matrix is used for many beamforming and DOA estimation algorithms such as MUSIC and MVDR.

Array configuration affects the steering vector and dimension of the signal vector. The simplest and standard array geometry for smart antenna system is ULA, in which the elements are aligned along a straight line and with a uniform interelement spacing usually considered $d=$ $\lambda / 2$, where $\lambda$ denotes the wavelength of the received signal. For prevention of mutual coupling effects and the DOA estimation errors resulting from misplaced or unwanted peaks in the spectrum, $d=\lambda / 2$ is the optimum interelement spacing in ULA configuration.

The main advantage of utilizing ULA is its simplicity, excellent directivity, and generating the narrowest main lobe in a given direction in comparison with other array geometries. However, DOA estimation accuracy and resolution are low at endfire directions when ULA is considered [11]. Thus, it is desirable to develop linear array configurations which perform equally well for all azimuth directions while maintaining simplicity, directivity, and high resolution. In the following, 5 simple array geometries are proposed based on ULA and evaluated to improve the DOA estimation performance in endfire directions.

Figure 1 depicts the ULA configuration and proposed SAA geometries which contain 7 elements. As illustrated in this figure, two elements from both ends of the ULA are taken and placed in different situations in top and/or bottom of the proposed arrays. So, the number of elements is the same and thereby the complexity of the arrays is close. The interelement spacing between the adjacent elements is half wavelength. All linear arrays are assumed to be symmetric around the origin and the element number $(N)$ is assumed to be odd. Consequently, the manifold matrix of the array has dimensions of $N \times M$.

If $\mathbf{a}_{\text {ULA }}\left(\theta_{m}\right)$ represents the steering vector for each of the input signals on the linear array, then for the symmetrical

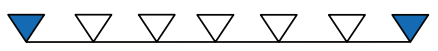

Uniform linear array (ULA)

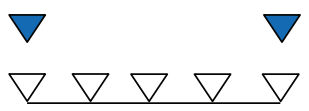

PA2

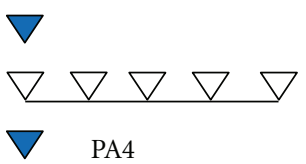

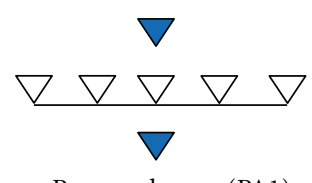

Proposed array (PA1)

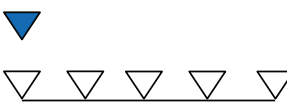

PA3

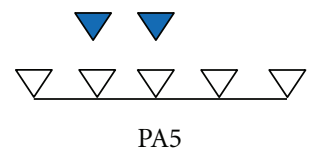

FiguRE 1: ULA and proposed array (PA) configurations.

linear array, $\mathbf{a}_{\mathrm{ULA}}\left(\theta_{m}\right)$ can be written as an $N \times 1$ vector as follows:

$$
\mathbf{a}_{\mathrm{ULA}}\left(\theta_{m}\right)=\left[\begin{array}{c}
e^{-j((N-1) / 2) k d \sin \theta_{m}} \\
e^{-j((N-3) / 2) k d \sin \theta_{m}} \\
\vdots \\
e^{+j((N-3) / 2) k d \sin \theta_{m}} \\
e^{+j((N-1) / 2) k d \sin \theta_{m}}
\end{array}\right],
$$

where $d$ is the interelement spacing and $k=2 \pi / \lambda$. For more details see Appendix A.

The steering vector for the first proposed SAA is denoted by $\mathbf{a}_{\mathrm{PAl}}\left(\theta_{m}\right)$ and can be written as the following.

$$
\mathbf{a}_{\mathrm{PAl}}\left(\theta_{m}\right)=\left[\begin{array}{c}
e^{-j((N-3) / 2) k d \sin \theta_{m}} \\
e^{-j((N-5) / 2) k d \sin \theta_{m}} \\
\vdots \\
e^{+j((N-5) / 2) k d \sin \theta_{m}} \\
e^{+j((N-3) / 2) k d \sin \theta_{m}} \\
e^{+j k d \cos \theta_{m}} \\
e^{-j k d \cos \theta_{m}}
\end{array}\right] .
$$

The first $N-2$ rows of $\mathbf{a}_{\mathrm{PAl}}\left(\theta_{m}\right)$ are related to the linear part of the array and the two remaining rows are obtained from the top and bottom displaced elements that are placed at the top and bottom of the array centre with the same interelement spacing in the proposed array. For more details see Appendix A.

The steering vector for other four configurations can be written similarly. The first $N-2$ rows are the same as both ULA and PA1 and the two later rows will differ as illustrated in Table 1. According to this table, it is seen that PA2 to PA4 and PA5 need two and one more multiplications rather than PA1 (and ULA), respectively. 
TABLE 1: The effect of dissimilar elements in steering vector for different proposed arrays.

\begin{tabular}{lcc}
\hline Proposed array & \multicolumn{1}{c}{ The effect of dissimilar elements 1 and 2 on steering vector } \\
\hline PA1 & $e^{+j k d \cos \theta_{m}}$ & $e^{-j k d \cos \theta_{m}}$ \\
PA2 & $e^{-j((N-3) / 2) k d \sin \theta_{m}} \times e^{+j k d \cos \theta_{m}}$ & $e^{+j((N-3) / 2) k d \sin \theta_{m}} \times e^{+j k d \cos \theta_{m}}$ \\
PA3 & $e^{-j((N-3) / 2) k d \sin \theta_{m}} \times e^{+j k d \cos \theta_{m}}$ & $e^{+j((N-3) / 2) k d \sin \theta_{m}} \times e^{-j k d \cos \theta_{m}}$ \\
PA4 & $e^{-j((N-3) / 2) k d \sin \theta_{m}} \times e^{+j k d \cos \theta_{m}}$ & $e^{-j((N-3) / 2) k d \sin \theta_{m}} \times e^{-j k d \cos \theta_{m}}$ \\
PA5 & $e^{+j k d \cos \theta_{m}}$ & $e^{-j k d \sin \theta_{m}} \times e^{+j k d \cos \theta_{m}}$ \\
\hline
\end{tabular}

\section{DOA Estimation Algorithms}

A variety of DOA estimation techniques are available which differ in modelling approach, computational complexity, resolution threshold, and accuracy $[4,5]$. In general, DOA estimation algorithms can be categorized as follows: conventional, subspace-based, maximum likelihood (ML)based, and subspace fitting techniques. Conventional and subspace-based methods are spectral-based methods, which rely on calculating the spatial spectrum of the received signal and finding the DOAs as the location of peaks in the spectrum. These methods are easy to apply and need fewer computations than the parametric methods. ML-based and subspace fitting techniques are parametric methods that directly estimate the DOAs without initially calculating the spectrum. Parametric algorithms have higher performance in terms of accuracy and resolution but the cost for this performance is higher complexity and more computations [5]. Performance of DOA estimation methods depend on the following:

(i) the type of incident signals, uncorrelated or coherent sources,

(ii) frequency bandwidth, narrowband or wideband,

(iii) the array geometry, 1D or 2D and uniform or nonuniform,

(iv) the type of noise, white or colored.

In this paper, two well-known spectral-based algorithms, MVDR and MUSIC, are used for evaluation of the proposed array geometries. The main goal of this paper is to show that the new geometry offers higher performance in DOA estimation, especially in endfire directions. Therefore, two DOA estimation methods are examined, one belongs to classic and the other one belongs to subspace-based methods.

3.1. Music Algorithm. MUSIC is a subspace-based algorithm. The eigenvectors of the covariance matrix belong to either two orthogonal signal or noise subspaces. If $M$ signals arrive on the array, the $N$ eigenvectors associated with $M$ larger eigenvalues of the covariance matrix span the signal subspace and the $N-M$ eigenvectors corresponding to the $N-M$ smaller eigenvalues of the covariance matrix span the noise subspace. The $M$ steering vectors that form the manifold matrix $\mathbf{A}(\theta)$ are orthogonal to the noise subspace and so the steering vectors lie in the signal subspace.

MUSIC algorithm estimates the noise subspace using eigendecomposition of the sample covariance matrix and then the estimate of DOAs is taken as those $\theta$ that give the smallest value of $\mathbf{A}^{H}(\theta) \mathbf{V}_{\mathbf{n}}$, where $\mathbf{V}_{\mathbf{n}}$ denotes the matrix of eigenvectors corresponding to the noise subspace. These values of $\theta$ result in a steering vector farthest away from the noise subspace and as orthogonal to the noise subspace as possible $[4,5]$. This is done by finding the $M$ peaks in the MUSIC spectrum defined by

$$
P_{\text {MUSIC }}(\theta)=\frac{1}{\mathbf{A}^{H}(\theta) \mathbf{V}_{\mathbf{n}} \mathbf{V}_{\mathbf{n}}^{H} \mathbf{A}(\theta)} .
$$

Several parameters such as the number of samples (snapshots), the number of elements, and the SNR affect the resolution threshold of the MUSIC.

3.2. MVDR Algorithm. In MVDR approach, it is attempted to minimize the power contributed by noise and undesired interferences, while maintaining a fixed gain in the look direction, usually equal to unity. This is written as

$$
\min E\left[|y(\theta)|^{2}\right]=\min \mathbf{w}^{H} \mathbf{R}_{\mathbf{x x}} \mathbf{w}, \quad \mathbf{w}^{H} \mathbf{A}\left(\theta_{0}\right)=1 .
$$

Using Lagrange multiplier, the weight vector that solves this equation is given by (10) as follows:

$$
\mathbf{w}=\frac{\mathbf{R}_{\mathbf{X X}}^{-1} \mathbf{A}(\theta)}{\mathbf{A}^{H}(\theta) \mathbf{R}_{\mathbf{X X}}^{-1} \mathbf{A}(\theta)} .
$$

The MVDR angular spectrum is defined by (11). The peaks in the MVDR spectrum occur whenever the steering vector is orthogonal to the noise subspace, so the DOAs are estimated by detecting the peaks $[4,17]$ as follows:

$$
P_{\mathrm{MVDR}}=\frac{1}{\mathbf{A}^{H}(\theta) \mathbf{R}_{\mathbf{x x}}^{-1} \mathbf{A}(\theta)} .
$$

\section{Performance Evaluation of Shirvani-Akbari Arrays}

In this section, the proposed SAA configurations are evaluated for source localization in different directions. The PA1 is previously proposed and investigated in [18, 19] with two extra elements with respect to the ULA. It has presented identical resolution threshold with the ULA in boresight directions where ULA has a high resolution. It has also presented higher resolution threshold in endfire directions. In addition, DOA estimation performance of the PA1 geometry is compared with two well-known 2D-array 


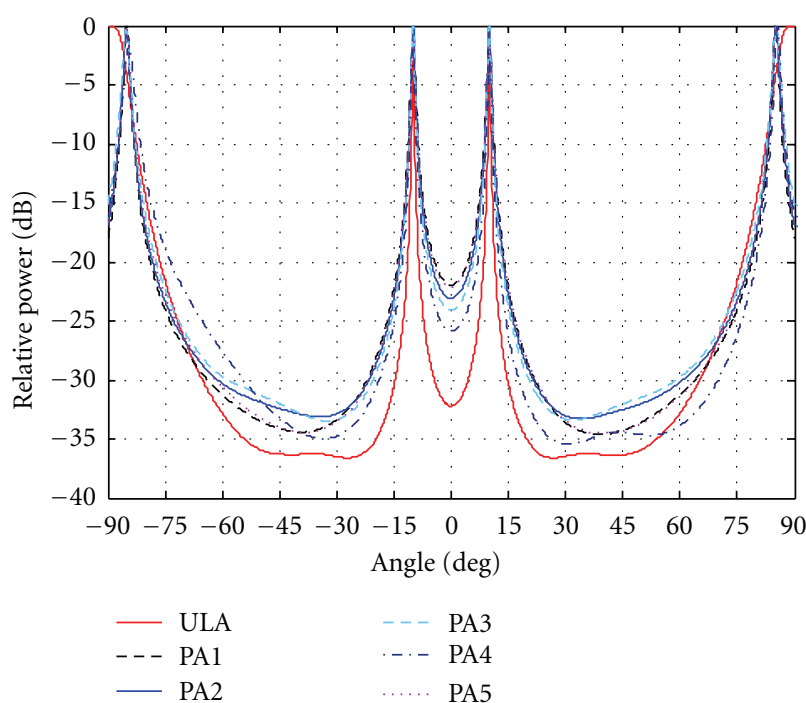

Figure 2: The MUSIC spectrum for the ULA and different PA configurations, DOAs $\left(-85^{\circ},-10^{\circ}, 10^{\circ}, 85^{\circ}\right)$.

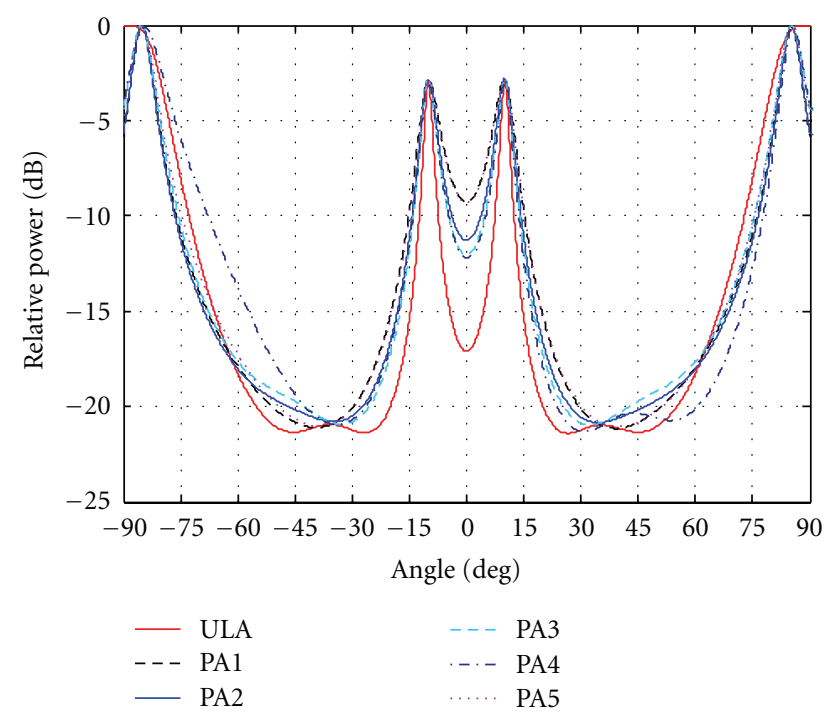

Figure 3: The MVDR spectrum for the ULA and different PA configurations, DOAs $\left(-85^{\circ},-10^{\circ}, 10^{\circ}, 85^{\circ}\right)$.

geometries: L-shaped and V-shaped in [20], and acceptable results are obtained with equivalent or lower complexity. Hence, in this section, different proposed SAAs in various conditions are compared to ULA.

In order to evaluate the performance of proposed SAAs, six arrays consisting of 7 elements with the linear and proposed geometries and the interelement spacing of half wavelength, are considered. The SNR is chosen to be $10 \mathrm{~dB}$ and the number of data snapshots is $K=100$. Four incident narrowband uncorrelated signal sources arriving at the angles $\left(-85^{\circ},-10^{\circ}, 10^{\circ}, 85^{\circ}\right)$ are assumed at both boresight and endfire directions.

Figure 2 shows the MUSIC spectrum for different configurations depicted in Figure 1. Simulation results show

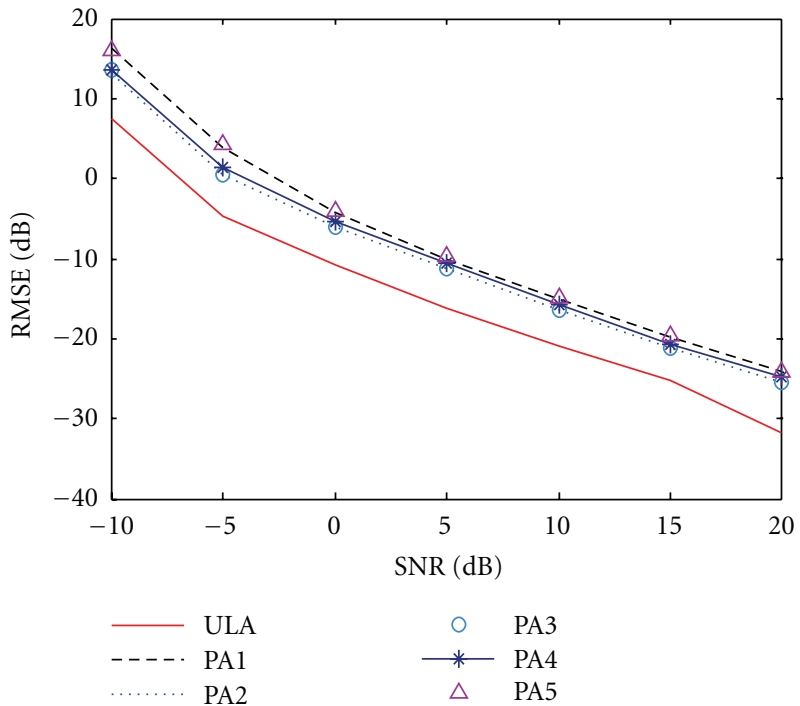

FIgURE 4: Total RMSE of the MUSIC algorithm for different configurations where signals are arriving at boresight directions.

that in spite of the conventional ULA, all of the proposed geometries are capable of detecting the signals arriving at the border of the spectrum and endfire directions, while they can also resolve other sources located at the middle of the spectrum. The MVDR spectrum for different arrays is shown in Figure 3. DOA estimation results using the MVDR method confirm the results of the MUSIC algorithm. As shown in these figures, the ULA has detected the signal sources arriving at $\left(-10^{\circ}, 10^{\circ}\right)$ successfully, but for the sources located at $\left(-85^{\circ}, 85^{\circ}\right)$, ULA is not capable to form the angular spectrum peaks, precisely. It means all of the proposed SAAs perform better than the conventional ULA at endfires.

To compare the DOA estimation accuracy of the proposed array geometries and the ULA, the total RMSE is calculated for different arrays in three cases of narrowband signal sources arriving at boresight directions, endfire directions, and a combination of these two cases. All of the arrays are considered with 7 elements as depicted in Figure $1 . K=100$ snapshots are used to estimate the array covariance matrix, and 1000 independent Monte-Carlo runs are performed to estimate the RMSE of the DOA estimates.

At first, two signal sources are assumed at $\left(-10^{\circ}, 10^{\circ}\right)$. Figures 4 and 5 represent the total RMSE for the ULA and other five configurations in different SNRs by using the MUSIC and MVDR algorithms for DOA estimation, respectively. The ULA represents lower values of RMSE at boresight directions in comparison with other configurations in different values of SNR.

In the second experiment, two signal sources are assumed at $\left(-85^{\circ}, 85^{\circ}\right)$, which are close to the array endfires. In this case, the total RMSE diagrams are observed in Figures 6 and 7, for the MUSIC and MVDR algorithms, respectively. At both of the algorithms, the total RMSE curve for the ULA is placed at a higher level than the other geometries. This can be interpreted as a weakness of the ULA at both ends of the array. 


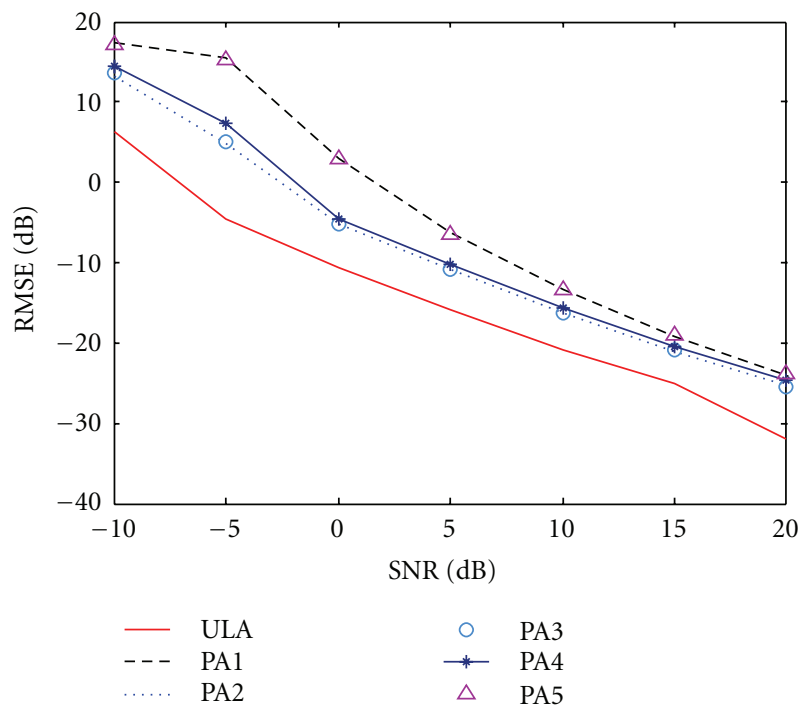

FIgure 5: Total RMSE of the MVDR algorithm for different configurations where signals are arriving at boresight directions.

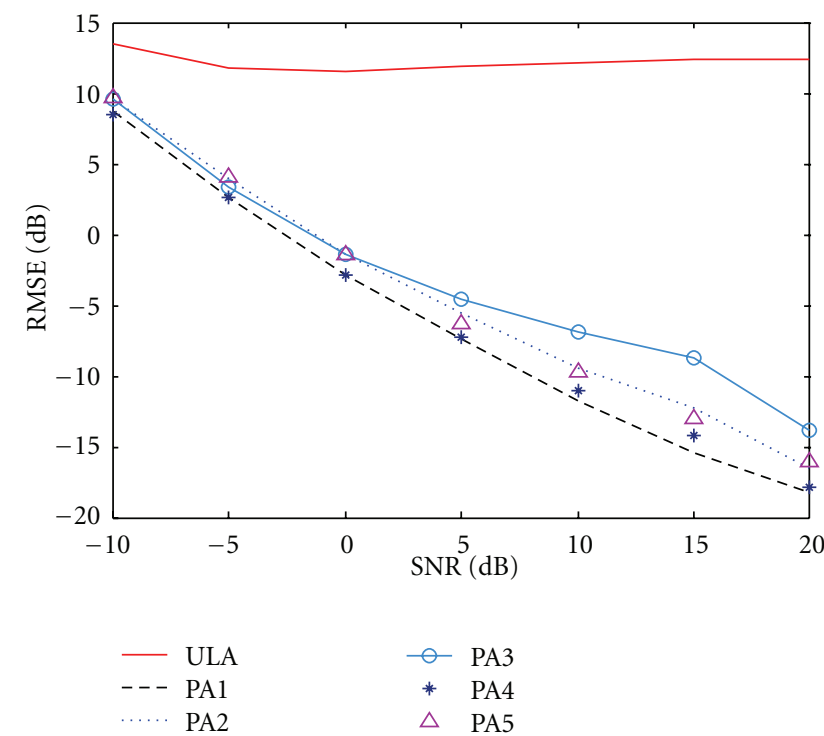

FIgURE 6: Total RMSE of the MUSIC algorithm for different configurations where signals are arriving at endfire directions.

To compare the DOA estimation accuracy of the proposed array geometries and the ULA in general, four incident narrowband signal sources arriving at angles $\left(-85^{\circ},-10^{\circ}, 10^{\circ}, 85^{\circ}\right)$ are considered, two of them are near to boresight and two others are near to endfire directions. The total RMSE curves are plotted in Figures 8 and 9 versus SNR for the MUSIC and MVDR, respectively. All of the proposed geometries represent better performance as lower total RMSE than the ULA. Even in higher SNRs, ULA has a high RMSE level.

The computational cost for all of the above configurations is almost identical because of equal element numbers and simplicity of array structure.

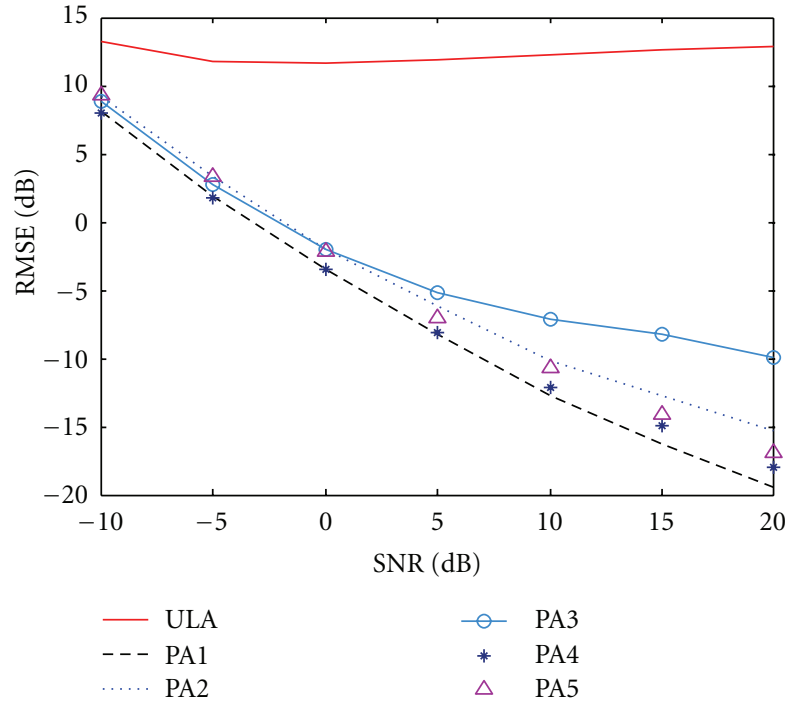

Figure 7: Total RMSE of the MVDR algorithm for different configurations where signals are arriving at endfire directions.

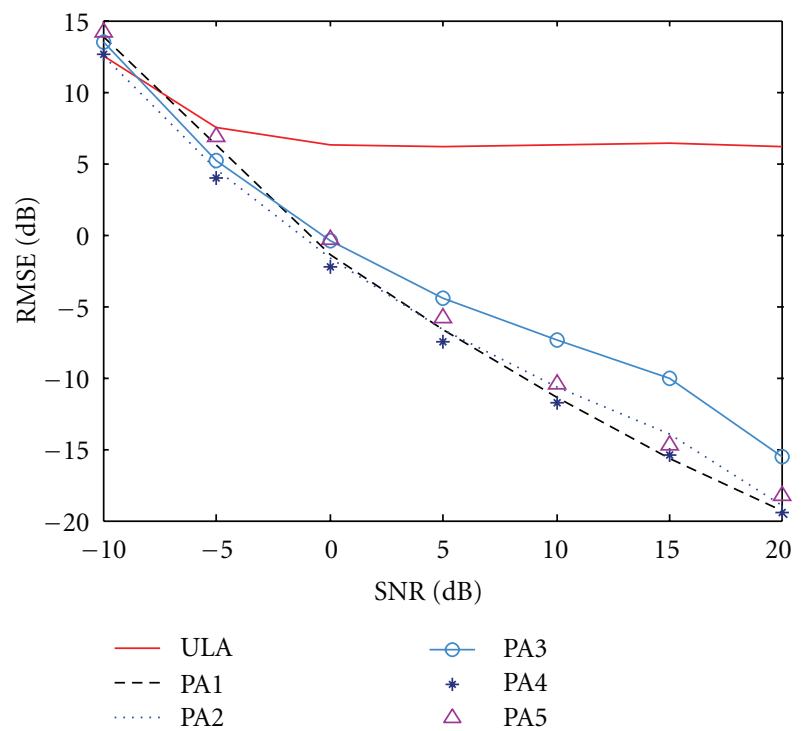

FIgURE 8: Total RMSE of the MUSIC algorithm for different configurations where signals are arriving at boresight and endfire directions.

\section{New Scenarios as a Combination of ULA and SAA}

To obtain the best performance for the array in terms of DOA estimation accuracy and computational load of the estimation process, a combination of the ULA and any of the proposed array configurations, namely, scenario 1 , can be employed. For investigation of the different arrangements, all of the array geometries shown in Figure 1 are experienced in this section. In order to calculate the MUSIC or MVDR spectrums at middle angles, $\left(-70^{\circ} \leq \theta \leq 70^{\circ}\right)$, the signals arrived at two extra elements of the proposed geometries are not considered and only the linear part of the proposed 


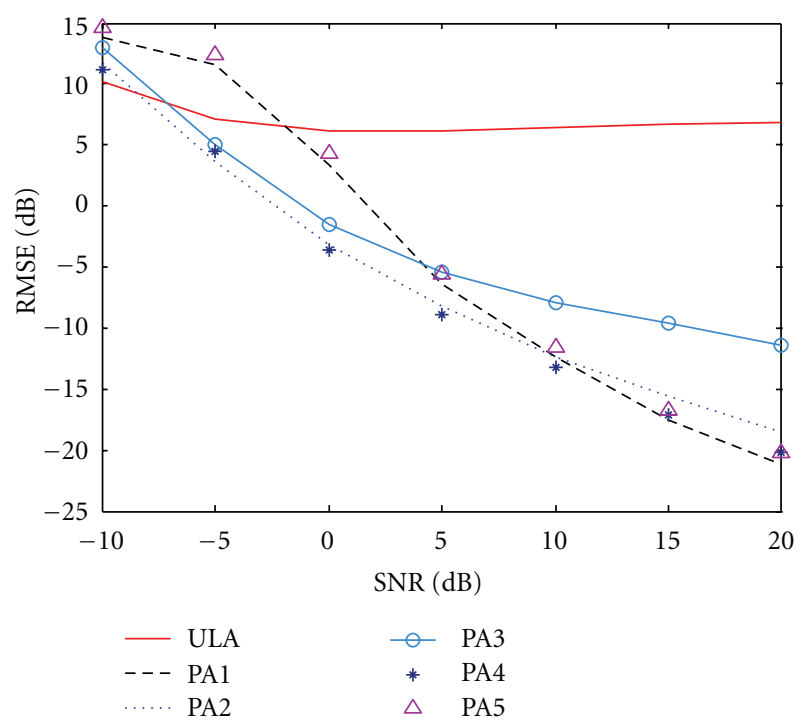

Figure 9: Total RMSE of the MVDR algorithm for different configurations where signals are arriving at boresight and endfire directions.

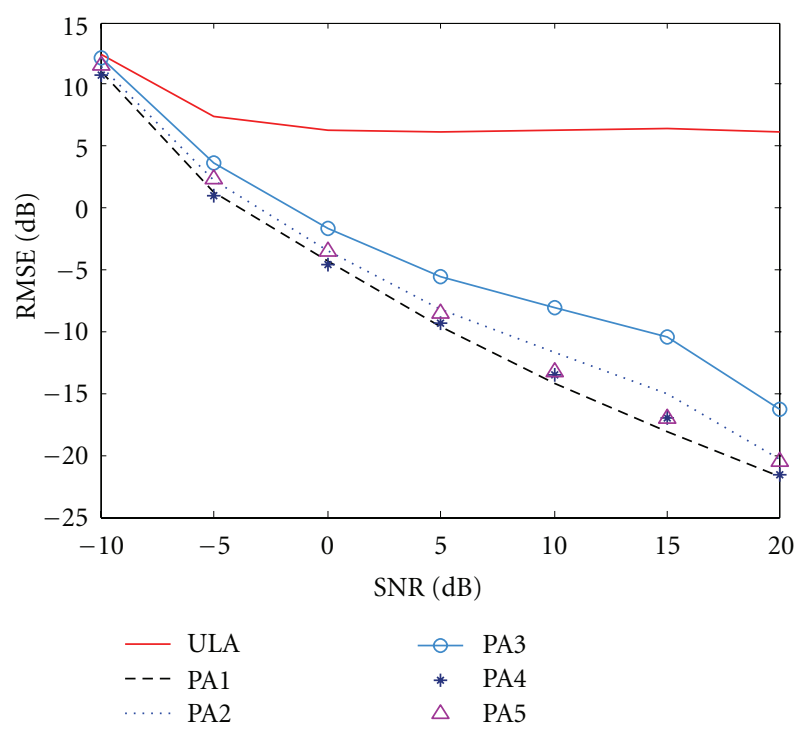

FIgUre 10: Total RMSE of the MUSIC algorithm for different combined array configurations in scenario 1.

array is used. For the endfire directions, $\left(-90^{\circ} \leq \theta \leq-70^{\circ}\right)$ and $\left(70^{\circ} \leq \theta \leq 90^{\circ}\right)$, the extra elements play an active role and their received signals are considered in spatial spectrum computation. Therefore, the advantages of the ULA and each of the proposed geometries can be jointly achieved with the least computational load.

Figures 10 and 11 depict the total RMSE of scenario 1, plotted in the case of four incident signals at boresight and endfire directions and with the described MUSIC and MVDR spectrum calculations. Lower total RMSE is observed in the lower SNRs for the proposed arrays in comparison with the ULA, using the combined array configuration. The computational cost is decreased too.

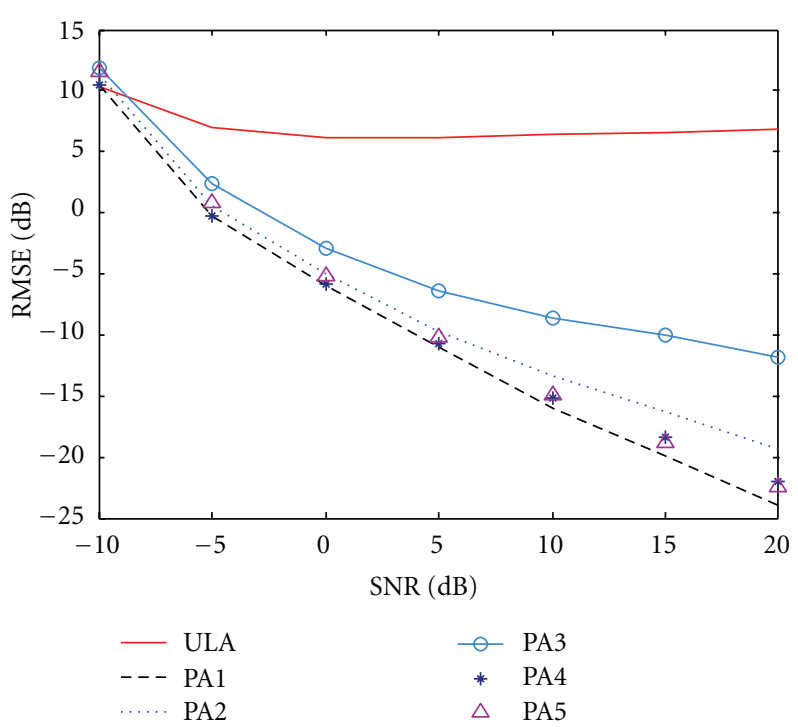

FIgURE 11: Total RMSE of the MVDR algorithm for different combined array configurations in scenario 1 .

Another proposed scenario is based on exploiting four extra elements in addition to the ULA with $N-2$ elements. This scenario can enhance the proposed array performance considering the number of elements and the complexity of the spectrum calculation in various situations including different DOAs and SNRs. For computation of the spectrum at middle angles $\left(-70^{\circ} \leq \theta \leq 70^{\circ}\right)$, the ULA elements and two ones at both ends of linear array are active without considering the remaining elements in the MUSIC or MVDR spectrum calculations. In computation of the spectrum at the endfire directions $\left(-90^{\circ} \leq \theta \leq-70^{\circ}\right)$ and $\left(70^{\circ} \leq \theta \leq\right.$ $90^{\circ}$ ), the two extra elements at both ends of linear part of the array are passive and the two other elements play an active role in calculation of the spectrum. As so, in the spectra computation, the array is considered with $\mathrm{N}$ elements and the computational load does not increase. Figures 12 and 13, respectively, show the total RMSE of the MUSIC and MVDR methods for scenario 2, where four signal sources are located at the above mentioned situations.

According to Figures 10 to 13, PA1 shows better performance with respect to other proposed configurations. Symmetrical structure of PA1 around both $\mathrm{x}$ and $\mathrm{y}$ axes provides a uniform view for the array. In the radiance pattern of PA1, as shown in Appendix B, better uniformity is seen over the whole space. It can be interpreted that the array has a uniform view over the space and consequently better performance than the other proposed configurations.

\section{Conclusion}

In this paper, 5 simple array configurations have been presented [that help us to estimate DOA of narrowband signal sources located at endfire directions that conventional ULA is not capable to accurately resolve these angles.] Two common DOA estimation algorithms, MUSIC and MVDR, are applied to compare the capability of arrays in finding 


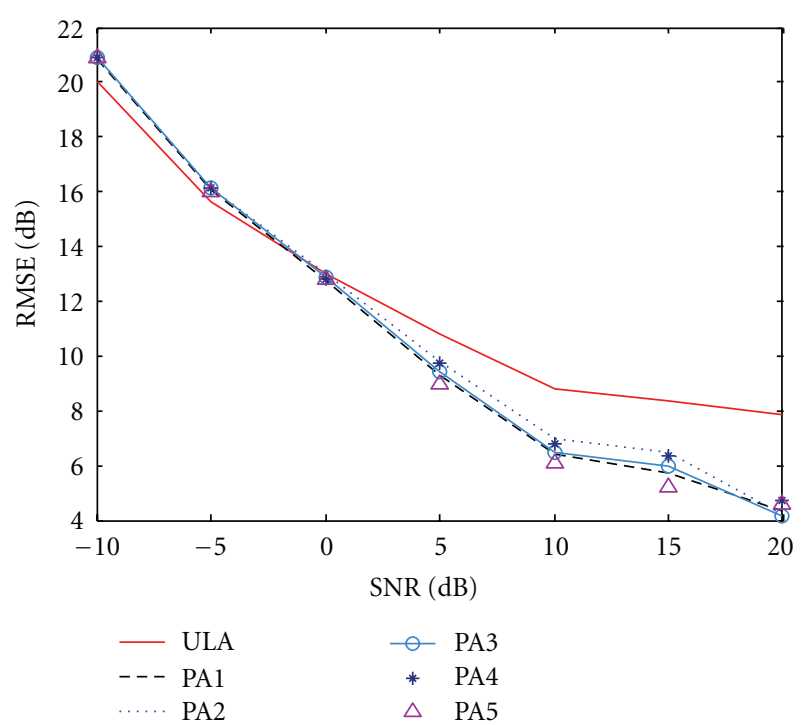

FIgURE 12: Total RMSE of the MUSIC algorithm for different combined array configurations in scenario 2.

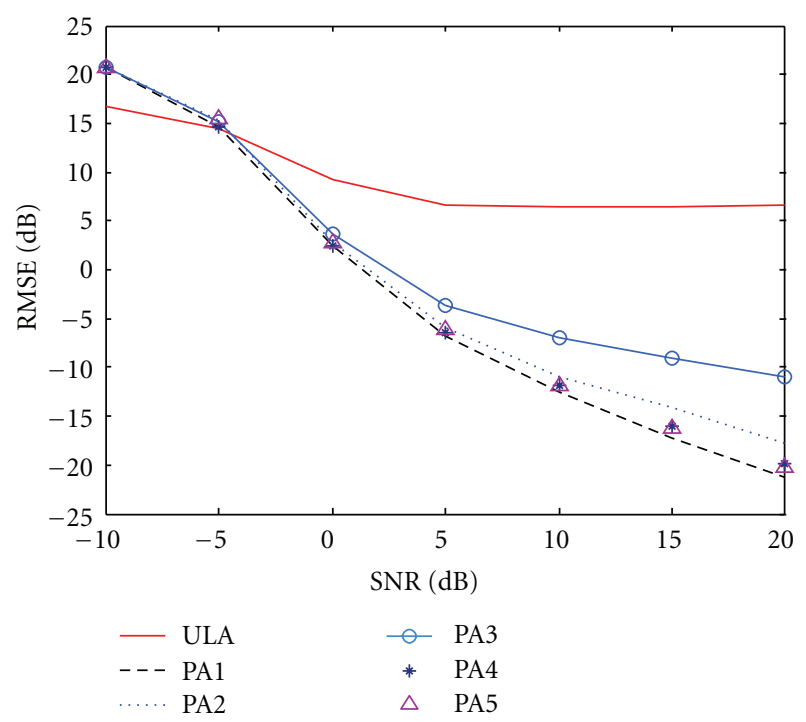

FIgURE 13: Total RMSE of the MVDR algorithm for different combined array configurations in scenario 2.

and resolving narrowband signal sources. Simulation results show better performance of the proposed SAAs in detection of signal sources located at endfire directions with accurate peaks generated at the spatial spectrum and consequently lower RMSE for calculated DOAs. At boresight directions, the proposed arrays present satisfying performance and in general the total RMSE for signal sources located at different situations is lower than the ULA. So, using one of these new simple arrangements, an improvement in DOA estimation has been achieved with equivalent computational cost. Also, simulation results show that SAA has higher impact in MUSIC compared to MVDR.

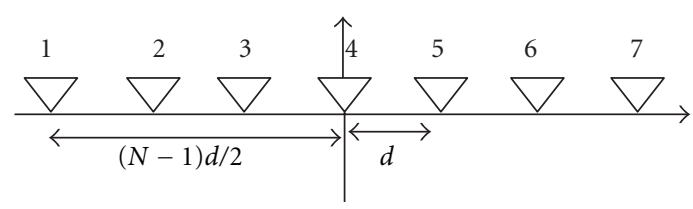

FIgURe 14: Symmetric ULA with 7 elements.

Also, in this paper, a novel combination of ULA and the proposed SAAs exploited to obtain the same performance with lower computational load. This efficient method is proposed in two scenarios and it is investigated to detect the narrowband sources. Simulation results show the better performance with respect to both conventional ULA and SAAs.

\section{Appendices}

\section{A.}

As depicted in Figure 14, the first element is placed in the distance of $(N-1) d / 2$ in the left side of the origin. The second one is placed in the distance of $(N-3) d / 2$ and so on. The $N$ th element is located in the distance of $(N-1) d / 2$ in the right side of the origin. For example, when $N=7$ the first element is in the distance of $(-3 d)$ of the origin and for the ULA with 7 elements the array vector will be expressed as (A.1) as follows:

$$
\mathbf{a}_{\mathrm{ULA}}\left(\theta_{m}\right)=\left[\begin{array}{c}
e^{-j 3 k d \sin \theta_{m}} \\
e^{-j 2 k d \sin \theta_{m}} \\
e^{-j k d \sin \theta_{m}} \\
1 \\
e^{+j k d \sin \theta_{m}} \\
e^{+j 2 k d \sin \theta_{m}} \\
e^{+j 3 k d \sin \theta_{m}}
\end{array}\right]
$$

This equation is generalized as (6) for the ULA with $\mathrm{N}$ elements. Rows of 1 to $N$ correspond to the first to $N$ th elements. The index of signals and weights in the associated signal and weight vectors corresponds to the index of array vector and therefore in the proposed arrays where the $(N-$ 1)th and $N$ th elements are assumed in above and/or below the array and no problem will occur in computations.

The array vector for the proposed arrays will be obtained in a similar way. Locations of the $(N-1)$ th and $N$ th elements cause different phases in $(N-1)$ th and $N$ th rows of array vector. For PA1, considering Figure 15, array vector is expressed as (7).

Also, for even number of array elements, origin will be considered between middle elements, $N / 2$ and $(N / 2+1)$ ones, in the case of symmetrical coordinate. In this case, the distance between middle elements to the array origin is $(-d / 2)$ and $(+d / 2)$, respectively.

It should be noted that the difference between symmetrical and nonsymmetrical coordinates is a constant phase. It means that adding a similar phase to all elements of steering vector has no effect on the results of DOA estimation and antenna beamforming. Moreover, the computational 


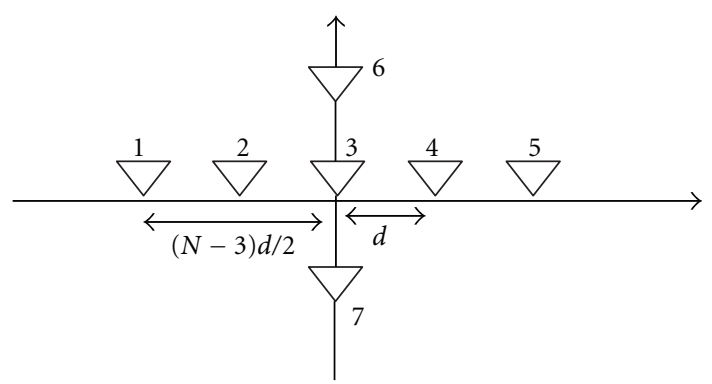

FIgURe 15: Symmetric PA1 with 7 elements.
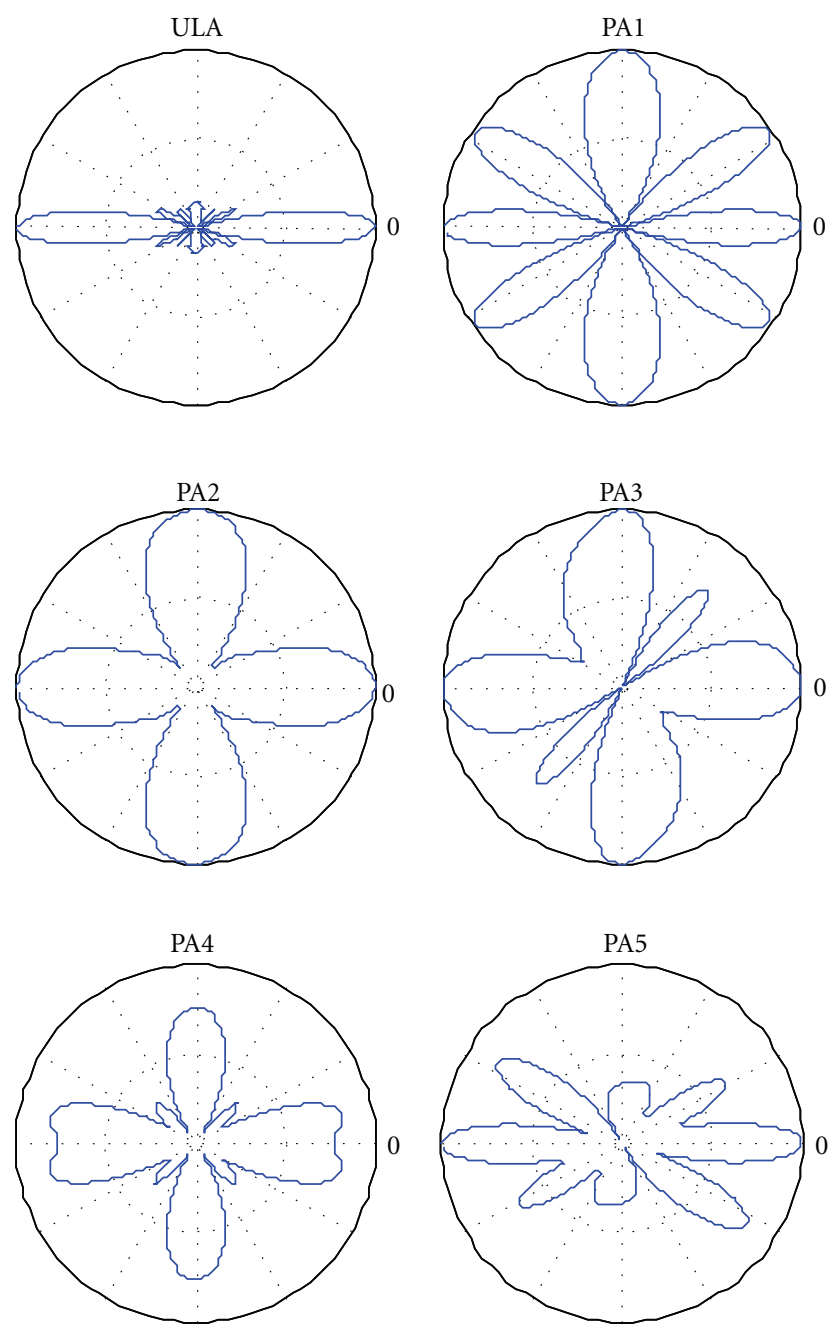

FIgURE 16: Array factor for ULA and proposed configurations.

complexity considering symmetrical or nonsymmetrical coordinates is the same. For example, absolute value of array factor for both coordinates is equal because constant phase has no effect in absolute value.

\section{B.}

To study the radiance behavior of the proposed configurations, the array factor is obtained for different arrays discussed in the paper. These diagrams are demonstrated in Figure 16. Comparing the array factor of different configurations shows that ULA has maximum radiance in the direction of array boresight (e.g., $0^{\circ}, 180^{\circ}$ ). Small lobes in other directions can be evaluated as the limitation of ULA in coverage of the whole space $\left(0^{\circ} \sim 360^{\circ}\right)$ which is the result of hidden elements. In array factor of the proposed configurations, better coverage is observed that can be the effect of the combination of two or three linear arrays with angle of $90^{\circ}$. This combination reduces the effect of hidden elements and therefore the proposed arrays can detect all the incident waves.

In other words, the alignment of array elements along one axis leads to the disadvantage of ULA. By adding another axis to the array, the limitation is removed. To remove the hidden elements effect in the planar array, array elements can be displaced in the distance of $d<\lambda / 2$ but this work causes coupling effect which is not desired. Therefore, the proposed array configurations are in accordance with Figure 1.

Also, ULA and PA1 are examined considering least mean square (LMS) and normalized LMS (NLMS) beamforming algorithms in [21]. Considering array factor, mean square error (MSE) and bit error rate (BER) metrics, simulation results show improved convergence speed and higher data transmission accuracy in different signal source locations and boresight angles as well as endfire ones, for proposed arrays with respect to ULA [21].

\section{Acknowledgment}

The authors would like to thank the editor and anonymous reviewers of the International Journal of Antennas and Propagation (IJAP) for their very helpful comments and suggestions which have improved the presentation of the paper.

\section{References}

[1] L. C. Godara, "Application of antenna arrays to mobile communications, part II: beam-forming and direction-ofarrival considerations," Proceedings of the IEEE, vol. 85, no. 8, pp. 1195-1245, 1997.

[2] F. Gross, Smart Antennas for Wireless Communications with MATLAB, McGraw Hill, New York, NY, USA, 2005.

[3] S. Shirvani-Moghaddam and M. Shirvani-Moghaddam, "A comprehensive survey on antenna array signal processing," Journal of Trends in Applied Sciences Research, vol. 6, no. 6, pp. 507-536, 2011.

[4] J. Foutz, A. Spanias, and M. K. Banavar, Narrowband Direction of Arrival Estimation for Antenna Arrays, Morgan \& Claypool, Ontario, Canada, 2008.

[5] L.C. Godara, Handbook of Antennas in Wireless Communications, CRC Press, Boca Raton, Fla, USA, 2002.

[6] E. Tuncer and B. Friedlander, Classical and Modern Directionof-Arrival Estimation, Academic Press, New York, NY, USA, 2009.

[7] P. Ioannides and C. A. Balanis, "Uniform circular arrays for smart antennas," IEEE Antennas and Propagation Magazine, vol. 47, no. 4, pp. 192-206, 2005. 
[8] M. Dessouky, H. Sharshar, and Y. Albagory, "Efficient sidelobe reduction technique for small-sized concentric circular arrays," Progress in Electromagnetics Research, vol. 65, pp. 187200, 2006.

[9] F. Gozasht, G. R. Dadashzadeh, and S. Nikmehr, "A comprehensive performance study of circular and hexagonal array geometries in the lms algorithm for smart antenna applications," Progress in Electromagnetics Research, vol. 68, pp. 281-296, 2007.

[10] T. Xia, Y. Zheng, Q. Wan, and X. Wang, "Decoupled estimation of 2-D angles of arrival using two parallel uniform linear arrays," IEEE Transactions on Antennas and Propagation, vol. 55, no. 9, pp. 2627-2632, 2007.

[11] R. M. Shubair and R. S. Nuaimi, "Displaced sensor array for improved signal detection under grazing incidence conditions," Progress in Electromagnetics Research, vol. 79, pp. 427441, 2008.

[12] Y. Hua, T. K. Sarkar, and D. D. Weiner, "An L-shaped array for estimating 2-D directions of wave arrival," IEEE Transactions on Antennas and Propagation, vol. 39, no. 2, pp. 143-146, 1991.

[13] F. Harabi, H. Changuel, and A. Gharsallah, "Direction of arrival estimation method using a 2-L shape arrays antenna," Progress in Electromagnetics Research, vol. 69, pp. 145-160, 2007.

[14] L. Jin, L. Li, and H. Wang, "Investigation of different types of array structures for smart antennas," in Proceedings of the International Conference on Microwave and Millimeter Wave Technology (ICMMT '08), vol. 3, pp. 1160-1163, Nanjing, China, April 2008.

[15] D. T. Vu, R. Renaux, R. Boyer, and S. Marcos, "Performance analysis of 2D and 3D antenna arrays for source localization," in Proceedings of the European Signal Processing Conference, pp. 661-665, EURASIP, Aalborg, Denmark, August 2010.

[16] X. Yang, T. Long, and T. K. Sarkar, "Effect of geometry of planar antenna arrays on Cramer-Rao Bounds for DOA estimation," in Proceedings of the IEEE 10th International Conference on Signal Processing (ICSP '10), pp. 389-392, Beijing, China, October 2010.

[17] M. A. Al-Nuaimi, R. M. Shubair, and K. O. Al-Midfa, "Direction of arrival estimation in wireless mobile communications using minimum variance distortionless response," in Proceedings of the Second International Conference on Innovations in Information Technology (IIT '05), pp. 1-5, 2005.

[18] F. Akbari, S. Shirvani Moghaddam, and V. T. Vakili, "MUSIC and MVDR DOA estimation algorithms with higher resolution and accuracy," in Proceedings of the 5th International Symposium on Telecommunications (IST '10), pp. 76-81, Tehran, Iran, December 2010.

[19] S. Shirvani Moghaddam, F. Akbari, and V. T. Vakili, "A novel array geometry to improve DOA estimation of narrowband sources at the angles close to the array endfire," in Proceedings of the 19th Iranian Conference on Electrical Engineering (ICEE2011), pp. 1-6, Tehran, Iran, May 2011.

[20] S. Shirvani Moghaddam and F. Akbari, "A novel ULA-based geometry for improving AOA estimation," EURASIP Journal on Advances in Signal Processing, vol. 2011, no. 39, 2011.

[21] S. Shirvani Moghaddam and F. Akbari, "Improving LMS/ NLMS-based beamforming using Shirvani-Akbari array," American Journal of Signal Processing, vol. 2, no. 4, pp. 70-75, 2012. 

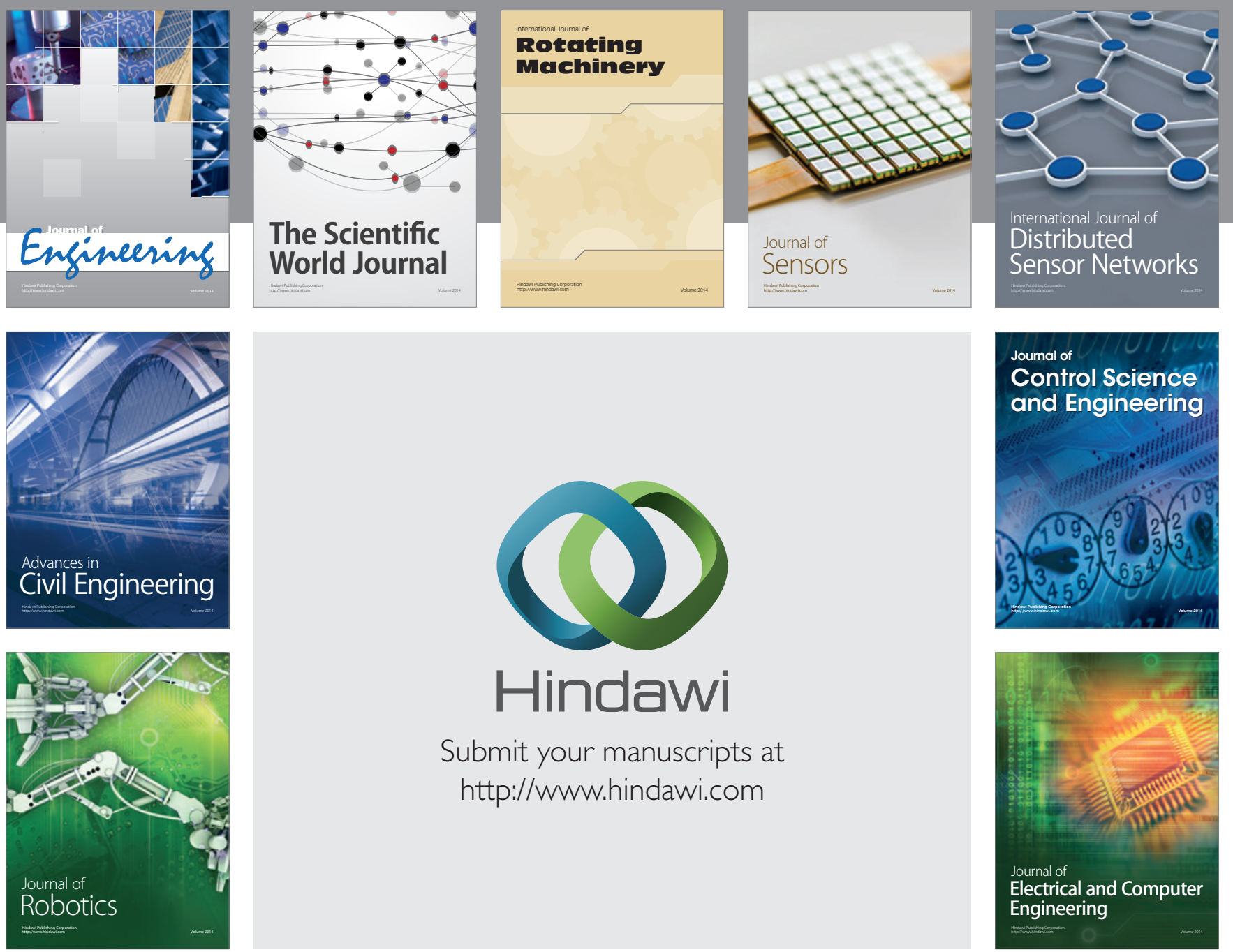

Submit your manuscripts at

http://www.hindawi.com
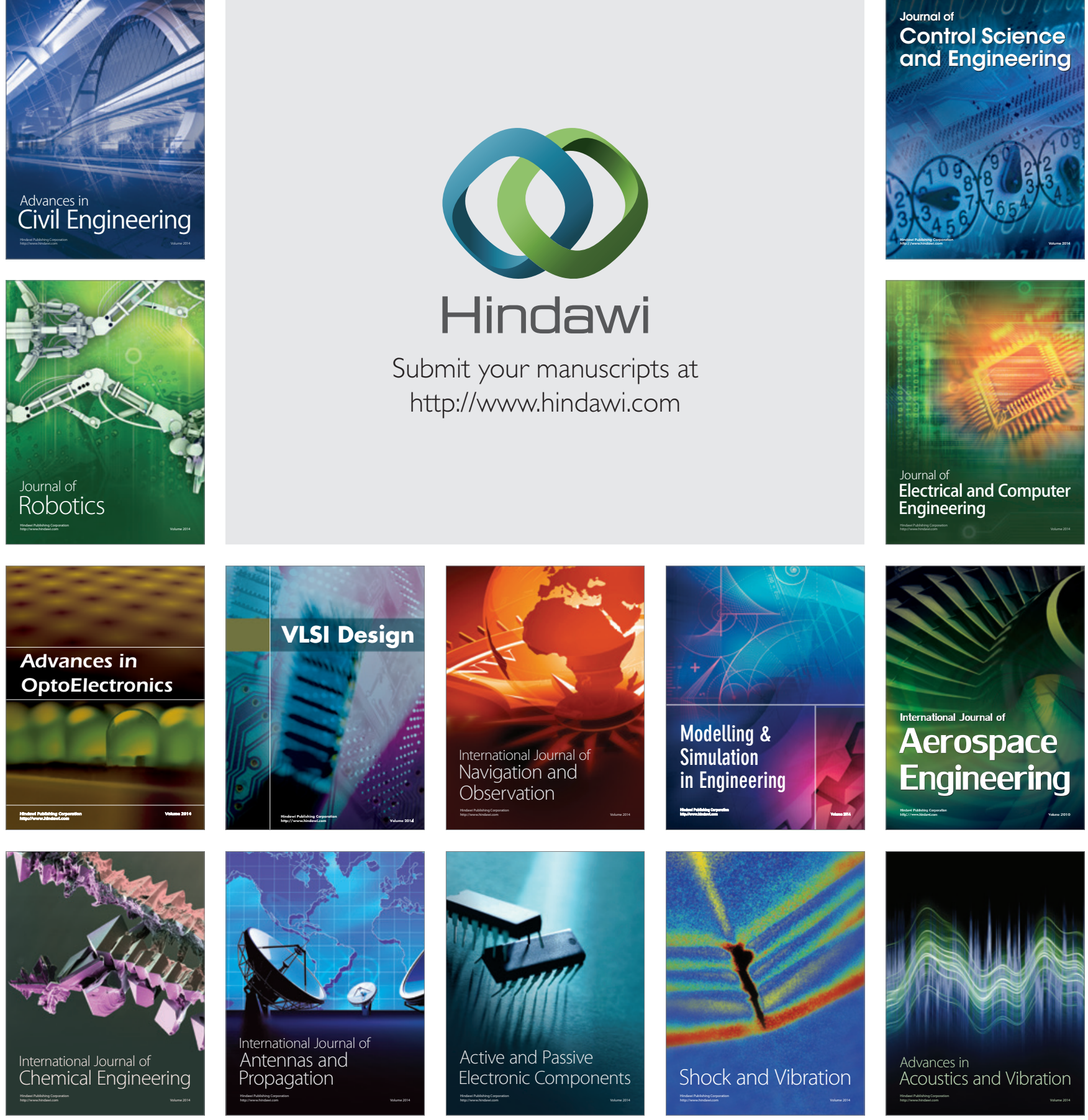